\title{
Thyroid Gland Involvement in Advanced Laryngeal Squamous Cell Carcinoma
}

\author{
Jan Warren A. Holgado, Precious Eunice R. Grullo, Jonel Donn Leo S. Gloria and Alfredo Quintin Y. Pontejos, Jr. \\ Department of Otorhinolaryngology, College of Medicine and Philippine General Hospital, University of the Philippines Manila
}

\begin{abstract}
Objective. This study aims to determine the prevalence and pattern of thyroid gland involvement in advanced laryngeal squamous cell carcinoma and to identify factors associated with its development.

Method. Records of 118 patients who underwent laryngectomy with thyroidectomy for advanced laryngeal squamous cell carcinoma in a tertiary government hospital from January 2010 to August 2015 were reviewed. Demographic, clinical and histopathology data were obtained. The relationship of the laterality of primary tumor bulk and lobe involvement was analyzed using Fischer's exact test. The association between thyroid gland invasion and other factors was analyzed using logistic regression.
\end{abstract}

Results. Thyroid gland involvement was found in $11 \%$ of the patients via direct spread in $92.3 \%$. Single lobe involvement accounted for $61.5 \%$ of cases. There is a tendency for laryngeal squamous cell carcinoma to invade the thyroid lobe ipsilateral to the primary tumor bulk. Extralaryngeal spread, tracheostomal involvement, and tracheal extension were associated with thyroid gland involvement.

Conclusion. Thyroid gland involvement in advanced laryngeal squamous cell carcinoma is rare; hence, indications for performing thyroidectomy in relation to the presence of thyroid gland involvement should be established to avoid unnecessary surgeries.

Key Words: laryngeal cancer, thyroidectomy, thyroid gland involvement

\section{Introduction}

Laryngeal cancer remains as a health burden in the Philippines with an incidence of 2.1 per 100,000 cases in 2012 making it the $4^{\text {th }}$ most common head and neck cancer among Filipinos behind thyroid, oral cavity, and

Presented at the Dr. Leon R. Lopez Annual Memorial Research Forum, December 3, 2015, Quezon City.

Corresponding author: Precious Eunice R. Grullo MD, MPH

Department of Otorhinolaryngology

2nd Floor, Ward 10

Philippine General Hospital

University of the Philippines Manila

Taft Avenue, Ermita, Manila 1000 Philippines

Telephone: +632 5548400 local 2151 or 2152

Email: prgrullo@up.edu.ph nasopharyngeal primary cancers, and $17^{\text {th }}$ overall for both sexes. ${ }^{1}$ Surgical resection, particularly total laryngectomy, has been established as the standard of management for laryngeal squamous cell carcinoma (squamous cell carcinoma) especially for advanced disease. ${ }^{2}$ Foreign and local guidelines state that routine thyroidectomy is performed in addition to laryngectomy particularly for advanced stages to achieve better oncologic margins due to its proximity to the larynx. . $^{2,3}$

However, several reports state that the incidence of thyroid gland involvement is low. ${ }^{4-6}$ In addition, there is an increased incidence of hypothyroidism in the post-operative period among laryngectomy patients especially when thyroidectomy is also performed., ${ }^{7,8}$ The role of routine thyroidectomy in laryngectomy for laryngeal squamous cell carcinoma is therefore controversial considering the low incidence of thyroid gland involvement and the increased incidence of post-operative hypothyroidism. In this period where accepted practice is shifting towards more conservative surgeries without sacrificing surgical outcome, selective thyroidectomy becomes an option.

This study determined the prevalence of thyroid gland involvement in advanced laryngeal squamous cell carcinoma and identified clinicopathologic factors associated with its occurrence to guide the surgical management of these cases.

\section{Materials and Methods}

This cross-sectional study was approved by the institutional review board. One hundred twenty five patients diagnosed with T3 and T4 laryngeal squamous cell carcinoma underwent surgery from January 2010 to August 2015 in the Department of Otorhinolaryngology of a tertiary hospital. One hundred eighteen patients underwent thyroidectomy and were included in the study. Thyroidectomy was done for these patients due to thyroid involvement based on pre-operative radiologic findings and/or intraoperative findings. Histopathology specimens were reviewed for the presence of thyroid gland involvement pathologic tumor stage ( $\mathrm{T}$ staging), tumor differentiation, tumor site, tumor extent, transcartilage invasion, extralaryngeal soft tissue extension, tracheal extension, and tracheostomal involvement. Demographic and clinicopathologic characteristics of these patients were obtained from the clinical charts. The relationship of the laterality of primary tumor bulk and lobe involvement was analyzed using Fischer's exact test. 
Thyroid Involvement in Laryngeal Squamous Carcinoma

Table 1. Univariate and Multivariate Regression Analysis of Factors Associated with Thyroid Gland Involvement

\begin{tabular}{|c|c|c|c|c|c|c|}
\hline & \multicolumn{3}{|c|}{ Univariate analysis } & \multicolumn{3}{|c|}{ Multivariate analysis } \\
\hline & OR & $C I$ & $p$ value & OR & $C I$ & p value \\
\hline Age (> 60 years) & 1.032 & $(0.960-1.110)$ & 0.390 & & -- & \\
\hline Tumor differentiation (moderate) & 1.010 & $(0.250-4.105)$ & 0.990 & 1.06 & $(0.186-6.055)$ & 0.946 \\
\hline Tumor differentiation (poor) & 3.650 & $(0.606-21.970)$ & 0.160 & 2.15 & $(0.104-44.283)$ & 0.621 \\
\hline Transcartilage invasion & 9.697 & $(2.041-46.071)$ & 0.002 & 1.01 & $(0.061-16.800)$ & 0.995 \\
\hline Extralaryngeal extension & 15.125 & $(3.155-72.519)$ & 0.000 & 17.90 & $(1.064-301.270)$ & 0.045 \\
\hline Tracheostomal involvement & 32.188 & $(5.370-192.150)$ & 0.000 & 11.46 & $(1.058-124.140)$ & 0.045 \\
\hline Tracheal extension & 8.750 & $(2.257-33.927)$ & 0.002 & 6.20 & $(0.766-50.208)$ & 0.087 \\
\hline
\end{tabular}

Table 2. Comparison of Studies on Thyroid Gland Involvement in Advanced Laryngeal Squamous Cell Carcinoma

\begin{tabular}{|c|c|c|c|c|}
\hline Author, Year & Number of cases & Incidence of TGI & Predictive factors & Route of spread \\
\hline Dadas et al., 2001 & 182 & $1.0 \%$ & Subglottic carcinoma & -- \\
\hline Gaillardin et al., 2012 & 87 & $12.6 \%$ & Subglottic extension $>10 \mathrm{~mm}$, cricoid cartilage invasion & Contiguous, non-contiguous \\
\hline Gurbuz et al., 2013 & 47 & $4.2 \%$ & $\begin{array}{l}\text { Subglottic extension }>10 \mathrm{~mm} \text {; invasion of paraglottic space, } \\
\text { thyroid cartilage, cricoid cartilage, and prelaryngeal tissue }\end{array}$ & Contiguous \\
\hline Dequanter et al., 2013 & 35 & $5.7 \%$ & Transglottic extent, subglottic extension $>10 \mathrm{~mm}$ & Contiguous \\
\hline Nayak et al., 2013 & 45 & $11.1 \%$ & Extralaryngeal soft tissue involvement, transglottic growth & Contiguous, non-contiguous \\
\hline Kumar et al.,2013 & 1,287 & $10.7 \%$ & Primary subglottic tumor, subglottic extension & -- \\
\hline Mourad et al., 2015 & 262 & $1.5 \%$ & Transglottic lesions, subglottic lesions & -- \\
\hline
\end{tabular}

The association between thyroid gland invasion and other factors was analyzed using logistic regression. All factors with $\mathrm{p}$ values less than or equal to 0.2 were included in the multivariate analysis using stepwise backward selection. All statistical analysis was done using Stata ver 13 (Statacorp. 2013. Stata statistical software: release 13. College station, tx: statacorp lp).

\section{Results}

Thyroid gland involvement was present in 13 patients (11.0\%). Direct spread was the more common route $(92.3 \%)$. Total thyroidectomy was performed in $74.8 \%$ of the patients. Among those who underwent total thyroidectomy, single lobe was involved in $61.5 \%$.

All patients with thyroid gland involvement had glottic tumors with transglottic extension. Well-differentiated squamous cell carcinoma accounted for $53.8 \%$ of cases. Presence of concurrent thyroid pathology was noted in 2 (15.4\%) patients, both of which were benign (colloid nodule, chronic lymphocytic thyroiditis). Statistical analysis of the relation of the laterality of primary tumor bulk and thyroid lobe involvement showed significant association ( $p=0.012)$.

Univariate analysis showed statistically significant association of transcartilage invasion, extralaryngeal spread, tracheostomal involvement, and tracheal extension with thyroid gland involvement (Table 1). After multivariate analysis, only extralaryngeal spread and tracheostomal involvement were associated with thyroid gland involvement. Although not statistically significant, there was an association between tracheal extension and thyroid gland involvement.

\section{Discussion}

The prevalence of thyroid gland involvement among advanced laryngeal squamous cell carcinoma patients who underwent laryngectomy with thyroidectomy was $11.0 \%$. This is comparable with the $1.5-12.6 \%$ reported in the literature (Table 2). ${ }^{4-7,9-12}$ On the other hand, thyroidectomy was done in more than $90.0 \%$ of patients with advanced laryngeal squamous cell carcinoma. This procedure however, is not without problematic complications in the long term follow up of patients. Hypothyroidism is a common sequela of total laryngectomy with an incidence of $20.0-47.4 \%$. The rate of post-laryngectomy hypothyroidism is further increased with inclusion of thyroidectomy. Alkan et al. reported the high incidence of hypothyroidism $(94.6 \%)$ following total laryngectomy with thyroidectomy. ${ }^{14}$ Despite preservation of residual gland, rate of post-operative hypothyroidism remained high $(22-88 \%)$ after total laryngectomy with hemithyroidectomy. $., 12,15-16$

Due to its proximity to the larynx, the thyroid gland may potentially be involved in advanced laryngeal cancers, particularly by the locally invasive squamous cell carcinoma. The route of spread to the thyroid gland by laryngeal cancer may course through two potential pathways: contiguous (direct invasion) and non-contiguous (lympho-vascular) spread. The more common route observed in the study was contiguous spread $(92.3 \%)$. This is consistent with previous reports, which showed the rate of direct invasion to the thyroid gland ranging from $60-100 \% .^{5-7,9}$ This can be explained by the close anatomical relationship of the thyroid gland with the larynx, only separated in some points by thin membranes and cartilages. Being a highly vascular organ surrounded by a rich network of lymphatics, the thyroid gland may also be invaded through lympho-vascular spread. One case in the study showed a small focus of squamous cell carcinoma within the thyroid gland with no evidence of direct extension from the primary tumor. This was associated with lymph node metastasis to the central group of neck nodes.

Predictors of thyroid gland involvement have been identified in several studies with subglottic extension, subglottic subsite, transglottic extent, transcartilage invasion, 
and extralaryngeal spread as consistent findings associated with thyroid gland involvement (Table 2). ${ }^{4-7,9-11,17}$ The study confirmed that extralaryngeal soft tissue spread was predictive of thyroid gland involvement. Tracheostomal involvement and tracheal extension were additional factors associated with thyroid gland involvement not previously reported in literature.

It has been hypothesized that laryngeal squamous cell carcinomas directly invading the thyroid gland reach the organ through regions of less resistance especially the cricothyroid membrane. Laryngeal carcinomas with transglottic extent grow laterally towards the paraglottic space down to the cricothyroid membrane, whereas primary subglottic cancers or those with subglottic extension invade directly through the cricothyroid membrane. Once the tumor reaches the extralaryngeal space, the thyroid gland becomes at risk of direct invasion. Tracheostomal involvement and tracheal extension, unique findings in the study, allow for thyroid gland involvement in a similar manner.

Tracheostomy is usually performed in patients who come in with severe dyspnea from airway obstruction by the primary tumor. As a surgical opening is introduced into the trachea, the tracheostoma may serve as a pathway through which direct spread of tumor from the lumen to the thyroid gland may occur. If the primary tumor has significant infraglottic growth during the time of tracheostomy, direct seeding becomes another route of spread to adjacent structures. Pre-laryngectomy tracheostomy, which becomes a risk factor for direct extension of tumor to the thyroid gland in relation to stomal involvement, should therefore be limited to cases where an emergent surgical airway needs to be established. Unfortunately, a major bulk of patients seen in our institution seeks consult at an advanced stage where tracheostomy cannot be avoided at times. However, there are cases where tracheostomy is performed to secure the airway for endoscopic laryngeal examination under general anesthesia. If intubation with smaller endotracheal tube can be done, tumor debulking becomes an option in such cases to avoid tracheostomy. This is possible provided that no delay in the planning of definitive management is to be encountered, as re-growth of the tumor will result to airway obstruction and eventual tracheostomy. Similarly, the delay between tracheostomy and laryngectomy adds to the risk of stomal involvement as this permits further infraglottic growth of the tumor.

The trachea composed of more elastic cartilaginous rings and intervening membranes compared to the laryngeal cartilages provides little resistance to invasive tumors. Only fascial planes separate the trachea from the thyroid gland. These allow for tumors with tracheal extension to directly invade the thyroid gland.

In summary, this study demonstrated that thyroid gland involvement in advanced laryngeal squamous cell carcinoma is associated with extralaryngeal soft tissue spread, tracheostomal involvement and tracheal extension. The tumor has a propensity to invade the thyroid lobe ipsilateral to the primary tumor bulk. Therefore, hemithyroidectomy (with isthmusectomy) is adequate for predominantly unilateral lesions in the presence of the above risk factors. Routine total thyroidectomy should be avoided and should be reserved for cases suspected of thyroid gland involvement with significant involvement of both laryngeal sides.

\section{References}

1. World Health Organization International Agency for Research on Cancer, Globocan 2012: Estimated cancer incidence, mortality and prevalence worldwide in 2012. Available from http://globocan.iarc.fr/ Pages/fact_sheets_cancer.aspx.

2. Pontejos AYP, Arquiza CJS. Manual for the management of head and neck malignancies, $2^{\text {nd }}$ ed. 2012. pp. 7-16.

3. National Comprehensive Cancer Network Clinical Practice Guidelines in Oncology, Head and Neck cancers, version i.2015.

4. Mendelsohn AA, Al-Khatib TA, Julien M, Payne RJ, Black MJ, Hier MP. Thyroid gland management in total laryngectomy: meta-analysis and surgical recommendations. Otolaryngol Head Neck Surg. 2009; 140(3):298-305.

5. Gürbüz MK, Açikalin M, Tasar S, et al. Clinical effectiveness of thyroidectomy on the management of locally advanced laryngeal cancer. Auris nasus larynx. 2014; 41(1):69-75.

6. Dequanter D, Shahla M, Paulus P, Vercruysse N, Lothaire P. The role of thyroidectomy in advanced laryngeal cancer and pharyngolaryngeal carcinoma. Indian J Otolaryngol Head Neck Surg. 2013; 65(2):181-3.

7. Nayak SP, Singh V, Dam A, et al. Mechanism of thyroid gland invasion in laryngeal cancer and indications for thyroidectomy. Indian J Otolaryngol Head Neck Surg. 2013; 65(Suppl 1):s69-s73.

8. Lo Galbo AM, Kuik DJ, Lips P, et al. A prospective longitudinal study on endocrine dysfunction following treatment of laryngeal or hypopharyngeal carcinoma. Oral Oncol. 2013; 49(9):950-5.

9. Gaillardin L, Beutter P, Cottier JP, Arbion F, Moriniere S. Thyroid gland invasion in laryngopharyngeal squamous cell carcinoma: prevalence, endoscopic, and CT predictors. Eur Ann Otorhinolaryngol Head Neck Dis. 2012; 129(1):1-5.

10. Razmpa E, Naghibzadeh B, Mehdizadeh J. Thyroid gland involvement in cancer of the larynx: a five-year multicenter study. Acta Med Iran. 2004; 42(3):181-4.

11. Kumar R, Drinnan M, Robinson M, et al. Thyroid gland invasion in total laryngectomy and total laryngopharyngectomy: a systematic review and meta-analysis of the English literature. Clin Otolaryngol. 2013; 38(5):372-8.

12. Mourad M, Saman M, Sawhney R, Ducic Y. Management of the thyroid gland during total laryngectomy in patients with laryngeal squamous cell carcinoma. Laryngoscope. 2015; 125(8):1835-8.

13. Biel MA, Maisel RH. Indications for performing hemithyroidectomy for tumours requiring total laryngectomy. Am J Surg. 1985; 150(4):435-9.

14. Alkan S, Baylancicek S, Ciftçic M, Sozen E, Dadaş B. Thyroid dysfunction after combined therapy for laryngeal cancer: a prospective study. Otolaryngol Head Neck Surg. 2008; 139(6):787-91.

15. Verloop H, Louwerens M, Schoones JW, Kievit J, Smit JW, Dekkers OM. Risk of hypothyroidism following hemithyroidectomy: systematic review and meta-analysis of prognostic studies. J Clin Endocrinol Metab. 2012; 97(7):2243-55.

16. Ortega-Gutierrez C, Luna-Ortiz K, Villavicencio-Valencia V, Herrera Gómez A, Téllez-Palacios D, Contreras-Buendía M. Hypothyroidism incidence after multimodal treatment for laryngeal cancer. Cir Cir. 2012; 80(5):448-50.

17. Dadas B, Uslu B, Cakir B, Ozdogan HC, Calis AB, Turgut S. Intraoperative management of the thyroid gland in laryngeal cancer surgery. J Otolaryngol. 2001; 30(3):179-83. 